\title{
The effect of home-based exercise in sleep quality and excessive daytime sleepiness in elderly people: A protocol of randomized controlled clinical trial
}

\author{
Glauber Sá Brandão1,2; Antônia Adones Callou²; Glaudson Sá Brandão3; Anderson Soares Silva4; Jessica Julioti Urbano4; \\ Newton Santos de Faria Junior5; Luis Vicente Franco Oliveira6; Aquiles Assunção Camelier,2.
}

\begin{abstract}
Background: Sleep-disordered breathing is associated with significant morbidity and mortality, presenting a high prevalence in the general population and being considered today as an important public health problem worldwide, affecting about $45 \%$ of the world population. It is estimated that about from 50 to $75 \%$ of the elderly complain of difficulties in initiating or maintaining sleep. The regular practice of physical exercise of mild to moderate intensity is associated with improved sleep quality in the elderly and has increased its recommendation as one of the main non-pharmacological resources of preventive and therapeutic action. Objectives: To verify the effect of a semi-supervised home-based physical exercise program on sleep quality in a sedentary elderly population, which influences the improvement of self-reported sleep quality and the reduction of excessive daytime sleepiness in a sedentary elderly population. Methods: This protocol study of randomized, controlled, blinded clinical trial was designed, is being conducted and will be reported according to the guidelines of the CONSORT (Consolidated Standards of Reporting Trials) Statement, and is being conducted from July 2016 to December 2017, involving elderly people of both sexes, over 60 years old, living in Senhor do Bonfim. The home physical exercise program is based on the recommendations of the American College of Sports Medicine for exercise and physical activity for the elderly. Results: The first expected outcome is the improvement of self-reported sleep quality verified through the PSQI. The secondary outcomes are reduction in excessive daytime sleepiness among the sedentary elderly subjects undergoing the semi-supervised home-based physical exercise program, verified by the ESS, and the adherence of the subjects to the program. Conclusions: We hope that through this study, we can show that the regular practice of semi-supervised home exercises is effective in improving sleep quality and reducing excessive daytime sleepiness in sedentary elderly people and can be considered as a therapeutic, safe, easy-to-implement, and low-cost non-pharmacological resource.
\end{abstract}

Key words: Exercise Training; Sleep; Quality of Life; Aged;

\section{INTRODUCTION}

Sleep-disordered breathing (SDB) is associated with significant morbidity and mortality, presenting a high prevalence in the general population and being considered today as an important public health problem worldwide, affecting about $45 \%$ of the world population ${ }^{(1)}$. In the study of Romano et al., the authors discuss the epidemiological and clinical characteristics of obstructive sleep apnoea (OSA), which has been shown to be a risk factor for serious health problems, such as hypertension, coronary artery disease, stroke, metabolic disorders, and excessive daytime

sleepiness ${ }^{(2)}$. The first epidemiological study describing sleep disorders was published in 1993, and showed that the prevalence of OSA ranged from $2 \%$ to $3 \%$ in women and $4 \%$ to $5 \%$ in men $^{(3)}$.

Punjabi in 2008, in a study involving young adults, showed that OSA was present in $3-7 \%$ of males and $2-5 \%$ of females ${ }^{(4)}$. With the improvement of diagnostic means, a recent survey conducted on a representative population of Sao Paulo City showed that $24.8 \%$ of men and $9.6 \%$ of women had OSA ${ }^{(5)}$. The high prevalence of SDB recorded in this population-based

TRIAL REGISTRATION: This protocol study has been approved by the Research Ethics Committee of the Escola Baiana de Medicina e Saúde Pública (Brazil), process no 39072514.6.0000.5544, and was registered on ensaiosclinicos.gov.br Identificator number: RBR-3cqzfy.

Corresponding Author: Glauber Sá Brandão. Rua Visconde do Rio Branco 162, Centro, Senhor do Bonfim, Ba, Brazil. CEP $48970-000$ e-mail: gbrandao@uneb.br Phone: 00557488487858

${ }^{1}$ Escola Baiana de Medicina e Saúde Pública, Salvador (BA), Brazil.

${ }^{2}$ Universidade do Estado da Bahia (UNEB), DEDC-VII, Senhor do Bonfim (BA) Brazil.

Full list of author information is available at the end of the article.

Financial support: This study was funded by the State University of Bahia, through the Support Program for the Training of Teachers and Administrative Technicians (PAC), without influencing any of the stages of the study, from the conception, development, analysis and interpretation of the results. LVFO receive grants Research Productivity, modality PQ1B; Process no. 313053 / 2014-6 of National Council for Scientific and Technological Development (local acronym CNPq), Brazil.

Submission date 20 February 2018; Acceptance date 02 April 2018; Publication date 11 April 2018 
sample might be attributed to the increased sensitivity of current recording techniques and scoring criteria.

A recent study, the HypnoLaus Sleep Cohort study, designed to assess the prevalence of SDB using state-of-theart polysomnographic recording techniques, showed that the prevalence of moderate to severe SDB ( $\geq 15$ events/h) was 23.4\% (95\% confidence interval, 20.9-26.0) in women and $49.7 \%(46.6-52.8)$ in men $^{(6)}$. These results suggest that SDB is highly prevalent with important public health outcomes, and that the definition and criteria of the gravity of the disorder should be revised.

It is estimated that about $50 \%$ of the elderly complain of difficulties in initiating or maintaining sleep. Sleep disturbances are commonly observed in this population because the aging process is associated with a growing prevalence of various comorbidities, considerable use of various medications, and psychosocial risk factors that compromise sleep and are associated with morbidity and mortality ${ }^{(7)}$. As most of the elderly present several factors from different domains that compromise their sleep, these complaints are more approached as a multifactorial geriatric health condition, causing the sleep disorders to be undiagnosed ${ }^{(8)}$.

The regular practice of physical exercise of mild to moderate intensity is associated with improved sleep quality in the elderly and has increased its recommendation as one of the main non-pharmacological resources of preventive and therapeutic action ${ }^{(9,10)}$. However, although scientific evidence demonstrates the benefits of regular physical exercise in the health and quality of life of the elderly, there is little adherence to this strategy. This low adherence may be correlated with external factors such as difficulty in locomotion or non-adaptation to the environment where the exercises are performed ${ }^{(11)}$.

Thus, our hypothesis is that the regular practice of home physical exercise is a safe, inexpensive, and easy-to-implement non-pharmacological therapeutic resource to improve sleep quality in the elderly.

\section{STUDY OBJECTIVES}

\section{Primary Objective}

- To verify the effect of a semi-supervised home-based physical exercise program on sleep quality in a sedentary elderly population.

\section{Secondary Objective}

- To verify the effect of excessive daytime sleepiness in sedentary elderly subjects of a semi-supervised home-based physical exercise program.
- To analyse the adherence of sedentary elderly subjects to the semi-supervised home-based physical exercise program.

\section{METHODS}

\section{Study design and setting}

This randomized, controlled, blinded clinical trial was designed, is being conducted and will be reported according to the guidelines of the CONSORT (Consolidated Standards of Reporting Trials) Statement ${ }^{(12)}$, shown in Figure 1. The study will use a convenience sample, composed of elderly subjects of a community in the municipality of Senhor do Bonfim, north-west of Brazil.

\section{Ethical and legal aspects}

This study was approved by the Research Ethics Committee Involving Human Beings of the Bahian School of Medicine and Public Health (protocol 39072514.6.0000.5544) and registered on ensaiosclinicos.gov.br (identification number RBR-3cqzfy). All participants have agreed and signed the informed consent form to be part of the study.

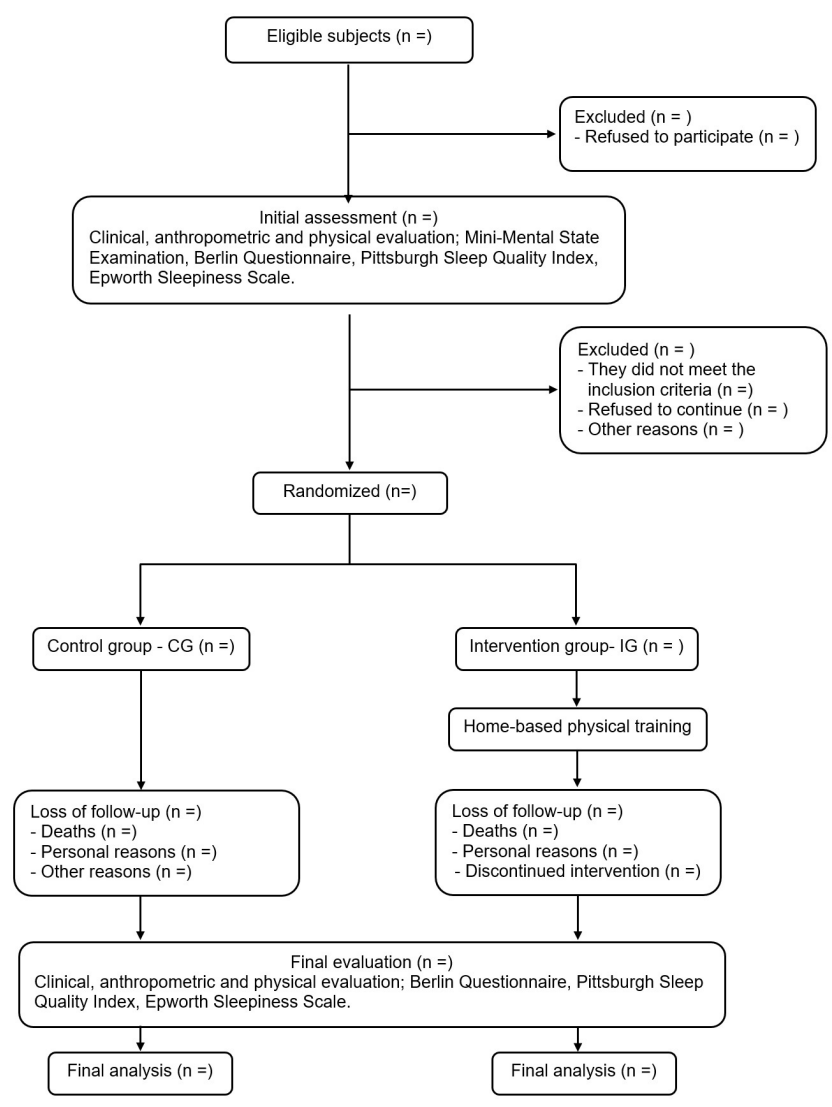

Figure 1. Flowchart of the study. 


\section{Subjects and recruitment procedure}

The research is being conducted from July 2016 to December 2017, involving elderly people of both sexes, over 60 years old, living in Senhor do Bonfim. Recruitment is taking place consecutively from throughout the community, initially by disseminating the conduct of research in local newspapers, radios, religious centres, elderly meeting groups, senior residency, neighbourhood association, and the senior citizen project developed by the city hall. A telephone number is provided so that the interested subjects can contact the research team.

\section{Eligibility criteria}

The inclusion criteria for the survey are as follows: have not performed regular exercise for at least 3 months prior to the start of the study, having a score $\geq 5$ on the Pittsburgh Sleep Quality Index (Brazilian Portuguese version; PSQI-BR) ${ }^{(13)}$, and not presenting a high risk for OSA verified through the Berlin Questionnaire ${ }^{(14)}$. Participants are excluded based on the following: having a cognitive deficit according to the Mini-Mental State Examination ${ }^{(15)}$, undergoing some treatment for sleep disorders (including the use of sleeping pills more than once a week), and having any clinical condition which contraindicates the performance of regular physical exercise, identified through a clinical and physiotherapeutic evaluation.

\section{Randomization}

After meeting the eligibility criteria, survey participants will receive a number in consecutive order of entry into the study and are randomly allocated to groups according to a random sequence generated by Research Randomizer. This randomization conceals the allocation to generate two groups: a control group (CG) and an intervention group (IG). All the elderly involved in the study participate in a 40-minute talk with explanations about the evaluation and intervention procedures.

The elderly of the IG will be informed to follow the guideline of home-based physical exercise program. To this end, they participate in theoretical-practical training for adequate accomplishment of the exercises and receive a booklet, developed by the researchers, that contains illustrative and written instructions on how to perform the exercises, besides a journal to register the frequency weekly.

The researchers, after making sure the subjects can perform all the exercises properly, guide the family members to help with and stimulate performance. Any change in the physical or mental condition of the subjects should be communicated by telephone to the research team. CG participants are informed that they should only continue their daily living activities.

\section{Clinical evaluation}

Evaluations are performed by a physician and previously trained physiotherapists. Research participants receive standardized verbal instructions on the procedures and are evaluated individually in the appropriate room. The entire evaluation process occurs before and after the intervention period. A clinical evaluation and a physical examination are performed, along with the collection of socioeconomic, demographic, and anthropometric data, and data on self-referred comorbidities, use of medications, sleep quality assessment, excessive daytime sleepiness, and OSA risk assessment.

Weight is measured with the subject wearing light clothing without shoes after emptying of the bladder, using a digital scale to the nearest $100 \mathrm{~g}$. Height is measured without shoes, with a stadiometer to the nearest $0.5 \mathrm{~cm}$ (model 200/5; Welmy Industria e Comercio Ltda, Sao Paulo, Brazil). BMI is calculated by dividing weight $(\mathrm{kg})$ by the square of height $(\mathrm{m})^{(16)}$.

\section{Pittsburgh Sleep Quality Index (PSQI)}

The PSQI, developed in 1989 by Buysse ${ }^{(17)}$ and validated for the Portuguese language by Bertolazi ${ }^{(13)}$, is used to verify sleep disorders and sleep quality by distinguishing good and bad sleepers. It consists of seven components, each punctuated on a scale of $0-3$. The components are subjective sleep quality, sleep latency, duration of sleep, habitual sleep efficiency, sleep disorders, use of medications for sleep, and daytime dysfunction. The scores of these components are summed to give an overall score ranging from 0 to 21 , with scores of $0-4$ indicating good sleep quality, 5-10 poor sleep quality, and $>10$ sleep disturbance ${ }^{(13)}$.

\section{Epworth Sleepiness Scale}

The Epworth Sleepiness Scale (ESS) is a self-administered scale that verifies the occurrence and intensity of excessive daytime sleepiness in eight situations of daily living. Participants are instructed to rate the likelihood of napping or falling a sleep in each of the eight specific situations on a score of $0-3$, with 0 : no chance of napping, 1 : small chance of napping, 2 : moderate chance of napping, and 3: strong chance of napping ${ }^{(18)}$.

\section{Berlin questionnaire}

The Berlin Questionnaire is a self-administered test to identify patients at high risk for respiratory sleep disorders in a variety of populations, with recognized efficacy particularly in distinguishing individuals at high risk for OSA. This test consists of 10 items organized into three categories: snoring and apnoea (5 items), daytime sleepiness (4 items), and systemic arterial hypertension and obesity ( 1 item). All marked responses are considered positive. Scores for each category can be positive or negative. Patients with positive scores in two or more categories are considered at high risk for OSA ${ }^{(14)}$.

\section{INTERVENTION:}

\section{Physical Exercise Program at Home}

The home physical exercise program is based on the recommendations of the American College of Sports Medicine for exercise and physical activity for the elderly ${ }^{(19)}$. The program 
is a combination of aerobic exercises, muscle strengthening exercises, balance training, motor coordination exercises, and flexibility exercises, prioritizing exercises involving large muscle groups. The program lasts for 12 consecutive weeks, with a minimum frequency of 3 sessions per week and a planned execution time of approximately $40 \mathrm{~min}$. During a session, two to three sets are performed with 5-15 repetitions for each exercise, at a target effort rate of 13-15 points ("a little difficult" to "difficult") on Borg's perceived exertion scale of 6-20(20).

The exercise program is performed individually at the home of each participant at his/her most convenient time, without direct supervision during its execution; however, on-site guidance is conducted through home visits every 15 days by a member of the research team. Participants are instructed to increase the intensity of the exercises, using Borg's scale as a parameter and in a manner proportional to their capacity of execution, evaluated by the research assistants in each of the visits.

The subjects perform the exercises using their own body weight and with the help of some low-cost equipment made by themselves (recyclable plastic bottles to demarcate the signage of the course, sticks and weights of 1 and $2 \mathrm{~kg}$ for performing the resistance exercises). The exercises performed are as follows:

- Warm-up exercises - Active free exercises of the upper and lower limbs, including extension, flexion, and rotation of the shoulders associated with breathing exercises.

- Aerobic exercises - Displacement of a stick with both hands, from the knees to above the head and returning to the knees, and exercises of ambulation with alternating flexion of the thighs and approaching the knee by hand on the opposite side.

- Resistance exercises - For the upper limbs: While standing with the elbow extended and the hand resting on the opposite thigh, the subject moves the whole hand diagonally upwards and then returns the hand to the thigh. For the lower limbs: For a squatting exercise, sitting on a chair with arms crossed in front of the body, the subject lifts to the orthostatic position and then returns to the sitting position.

- Balance and coordination exercises - The participant walks on a straight line and walks away from queued obstacles with progressively smaller distances. The exercise evolves as possible, and the walk is performed by placing the heel of one foot on the toes of the other foot (standing foot). Note: To ensure safety, these exercises are performed close to a fixed furniture in the house, making it possible to lean when needed.

- Stretching exercises - From a sitting position on bed and with the knees extended, the participant tries to reach the tip of the feet. From a sitting position on a chair and with the feet resting on the ground, the participant performs rotation of the trunk to one side and elevation of the upper limb, on the same side, above the head, stretching as high as possible.

During the period of the program (12 consecutive weeks), regular home visits are made to the participants of the two groups to clarify doubtsand encourage adherence to the program. The IG receives specific monitoring regarding the practice of the exercises and assistance to possible adverse events. After the 12-week period, the subjects in both groups are reassessed and encouraged to continue with the home program. Meanwhile, the subjects of the CG are followed up for the regular practice of the same home exercises performed by the IG participants in the same period. Adherence to the program is verified through weekly records that are filled in by the elderly with the help of his relatives and are also certified by the assistants during the home visits.

\section{Statistical analysis}

To detect whether randomization generated comparable groups, the characteristics of the two groups will be compared before the intervention using the Student's t-test for independent samples in relation to the numerical variables and the Pearson chi-square test for categorical variables. To test the normality of the data, a study of the histogram of the study, mean and median, standard deviation, skewness and kurtosis will be performed, and for its confirmation we will use the Shapiro-Wilk normality test. The significance level established for all analyzes will be $p<0.05$ and all statistical procedures will be analyzed and processed in the Statistical Package of Social Sciences SPSS 21.0 (IBM ${ }^{\circledR}$ SPSS version 21, IBM, Armonk, NY).

\section{Sample size}

The calculation of sample size was based on a previous study by Chen et al. ${ }^{(21)}$ that identified a significant improvement in overall sleep quality using the PSQI. Using a two-tailed alpha of 0.05 and $80 \%$ power to detect a moderate effect size of 0.63 (0.30-0.80), a sample size of 82 subjects was necessary to comprise our sample. Eight patients were added (20\%) to each group to allow for possible sample loss.

\section{Outcome measures}

The first expected outcome is the improvement of self-reported sleep quality verified through the PSQI. The secondary outcomes are reduction in excessive daytime sleepiness among the sedentary elderly subjects undergoing the semi-supervised home-based physical exercise program, verified by the ESS, and the adherence of the subjects to the program. 


\section{Assessor blinding:}

Researchers involved in the interpretation of clinical tests and the statistician responsible for data analysis will be blind to the composition of the study groups.

\section{Data monitoring and quality control:}

Systematic trainings exclusively for evaluations are carried out to 5 assistants, and 10 assistants are being used for home monitoring, 5 of whom are visiting the IG and the other 5 are visiting the CG. The research assistants ensure equal distribution of the number of subjects being evaluated and the number of homes being visited.

\section{RESULTS}

The results of this study are expected to show that sedentary elderly people with sleep disorders present a significant improvement in sleep quality and a reduction in excessive daytime sleepiness when participating in a semi-supervised physical exercise program. Such expected results are based on studies that have already demonstrated the effectiveness of physical exercise programs in improving sleep quality ${ }^{(9,22-24)}$ and reducing excessive daytime sleepiness in the elderly ${ }^{(25)}$.

\section{DISCUSSION}

However, most of these studies adopted professional supervision during the execution of exercise programs, as well as performance of the exercises in training and/or rehabilitation centres, which is a limiting factor for the participation of the elderly because they experience difficulties in relation to the transfer ${ }^{(24,26)}$. This study advocates a program of home physical exercise, supervised only every fortnight, easy to apply, and inexpensive. It has already been shown that physical exercise performed at home, besides being the preference of the elderly person, can present important health benefits ${ }^{(26-28)}$, demonstrating greater adherence and continuity after the end of the proposed program ${ }^{(29)}$.

Knowledge on the effects of home exercises on sleep quality of the elderly is still very incipient. A randomized trial similar to the one described here was conducted by Chen et al. ${ }^{(22)}$ The trial involved 56 elderly people (mean age, $71.7 \pm 8.1$ ), where the IG performed the Baduanjin exercise program in their households and the CG did not perform any specific activity. The IG subjects received a videotape and an educational booklet with pictures of how to perform the exercises, were instructed to perform 30 min of exercise thrice a week for 12 weeks, and received a telephone follow-up twice a week. After the proposed period, a significant difference was observed in the PSOI scores between the groups of subjects.

A secondary outcome of this study is reduction in excessive daytime sleepiness, which is currently recognized as an important public health problem ${ }^{(28)}$. It would be expected that elderly people who practice physical exercise present a reduction in excessive daytime sleepiness, as observed in a study by Li et al. ${ }^{(25)}$ using supervised Tai Chi exercises. Moreover, in a study by Chen et al. ${ }^{(24)}$, the practice of Yoga resulted in a significant reduction in daytime sleepiness in elderly people, verified through ESS.

In a recent randomized, controlled trial study, Lu et al. ${ }^{(30)}$, verified the effects of a 24-week Tai Ji Quan training program on sleep quality, quality of life, and physical performance among elderly Chinese women with knee osteoarthritis (OA). Subjects were randomly assigned to either a Tai Ji Quan group or a control group. Participants in the Tai Ji Quan group completed training sessions three times per week, while those in the control group had bi-weekly educational classes. The primary outcome was total score of the PSQI, and the secondary outcomes were seven subscales of the PSQl; physical component summary and mental component summary of the 36-item Short Form Health Survey (SF-36); Berg Balance Scale (BBS); and Timed Up and Go (TUG). Compared with the control group, participants in the Tai Ji Quan group had significantly improved primary outcome (PSQI score) and secondary outcomes, including three PSQI sub-scores (sleep latency, daytime total sleep, and SF- 36 PCS. The Tai Ji Quan group also had significant improvements compared to baseline in three PSQI sub-scores (sleep latency, sleep disturbance, sleep latency, BBS, and TUG). The authors concluded that Tai Ji Quan training is an effective treatment approach to improve sleep quality and quality of life in elderly Chinese women with knee OA.

One of the strengths of this study is that after random allocation of participants, both IG and CG periodically receive the same orientations and stimuli in relation to sleep hygiene measures, allowing them to present different behaviours only with regard to the practice of physical exercise, so that we can show differences between both groups. Some studies suggest that direct contact with the participants via telephone, Internet, or personal visit increases the adherence of elderly subjects to home exercise programs ${ }^{(29-32)}$.

We hope that through this study, we can show that the regular practice of semi-supervised home exercises is effective in improving sleep quality and reducing excessive daytime sleepiness in sedentary elderly people and can be considered as a therapeutic, safe, easy-to-implement, and low-cost non-pharmacological resource.

\section{Acknowledgements}

The authors are especially grateful to the Department of Education - campus VII of the State University of Bahia - UNEB for providing the necessary equipment for the evaluation of the participants, the members of the research group on quality of life and healthy aging - QUALES for the dedication in data collection and In the elderly during the whole period of the intervention. Special thanks to the elderly who accepted to participate in this study. 


\section{DESCRIPTION OF RISKS}

There will be no risks for included patients.

\section{AUTHORS CONTRIBUTIONS}

GSB and AAC: conception and design of this study; AAC, GSB and, AAC: data acquisition, draft and revision of the study; JJU, ASS, NSFS, LVFO, GSB and AAC: analysis and interpretation of data, draft and revision of the study; RSBR: All authors: final approval of the version to submission.

\section{CONFLICTS OF INTEREST}

The authors declare that they have no competing interests.

\section{AUTHOR DETAILS}

${ }^{3}$ Clínica de Diagnóstico e Especialidades (IMAIS), Senhor do Bonfim (BA), Brazil.

${ }^{4}$ Sleep Laboratory, Rehabilitation Sciences Master's and PhD Degree Program, Nove de Julho University (UNINOVE), Sao Paulo, (SP), Brazil.

${ }^{5}$ Physical Therapy Course, Universidade do Estado de Minas Gerais (UEMG), Divinópolis (MG), Brazil.

${ }^{6}$ Medical School, University Center of Anapolis (UNIEVANGELICA), Anápolis, (GO), Brazil.

\section{REFERENCES}

1. Stranges S, Tigbe W, Gomez-Olive FX, Thorogood M, Kandala NB. Sleep problems: an emerging global epidemic? Findings from the INDEPTH WHO-SAGE study among more than 40,000 older adults from 8 countries across Africa and Asia. Sleep. 2012;35(8):1173-1181. PMID: 22851813

2. Romano S, Salvaggio A, Hirata RP, Lo Bue A, Picciolo S, Oliveira LV, et al. Upper airway collapsibility evaluated by a negative expiratory pressure test in severe obstructive sleep apnea. Clinics (Sao Paulo). 2011;66(4):56772. PMID: 21655748

3. Young T, Palta M, Dempsey J, Skatrud J, Weber S, Badr S. The occurrence of sleep-disordered breathing among middle-aged adults. N Engl J Med. 1993;328:1230-5. PMID: 8464434

4. Punjabi NM. The epidemiology of adult obstructive sleep apnea. Proc Am Thorac Soc. 2008;5:136-43. PMID: 18250205

5. Tufik S, Silva RS, Taddei JA, Bittencourt LRA. Obstructive Sleep Apnea Syndrome in the São Paulo Epidemiologic Sleep Study. Sleep Medicine. 2010;11(5):441-6. PMID: 20362502

6. Heinzer R, Vat S, Marques-Vidal P, Marti-Soler H, Andries D, Tobback $\mathrm{N}$, et al. Prevalence of sleep-disordered breathing in the general population: the HypnoLaus study. Lancet Respir Med. 2015;3(4):310-318. PMID: 25682233

7. Miner B, Kryger MH. Sleep in the Aging Population. Sleep Med Clin. 2017;12(1):31-38. PMID: 28159095

8. Crowley K. Sleep and sleep disorders in older adults. Neuropsychol Rev. 2011;21(1):41-53. PMID: 21225347

9. Akbari Kamrani AA, Shams A, Shamsipour Dehkordi P, Mohajeri R. The effect of low and moderate intensity aerobic exercises on sleep quality in men older adults. Pakistan J Med Sci. 2014;30(2):417-21. PMID: 24772155

10. Du S, Dong J, Zhang H, Jin S, Xu G, Liu Z, et al. Taichi exercise for self-rated sleep quality in older people: A systematic review and meta-analysis. Int J Nurs Stud. 2015;52(1):368-79. PMID: 24934815

11. Gobbi S, Sebastião E, Papini CB, Nakamura PM, Valdanha Netto A, Gobbi LTB, et al. Physical Inactivity and Related Barriers: A Study in a Community Dwelling of Older Brazilians. J Aging Res. 2012;6:1-8. PMID: 23209906

12. Moher D, Hopewell S, Schulz KF, Montori V, Gøtzsche PC, Devereaux PJ, et al. CONSORT 2010 Explanation and Elaboration: updated guidelines for reporting parallel group randomised trials. Int J Surg. 2012;10(1):28-55. PMID: 22036893

13. Bertolazi AN, Fagondes SC, Hoff LS, Dartora EG, da Silva Miozzo IC, de Barba MEF, et al. Validation of the Brazilian Portuguese version of the Pittsburgh Sleep Quality Index. Sleep Med. 2011;12(1):70-5. PMID: 21145786
14. Vaz AP, Drummond M, Mota PC, Severo M, Almeida J, Winck JC. Tradução do Questionário de Berlim para língua Portuguesa e sua aplicação na identificação da SAOS numa consulta de patologia respiratória do sono. Rev Port Pneumol. 2011;17(2):59-65. DOI: 10.1016/S08732159(11)70015-0

15. Brucki SMD, Nitrin R, Caramelli P, Bertolucci PHF, Okamoto IH. Sugestões para o uso do mini-exame do estado mental no Brasil. Arq Neuropsiquiatr. 2003;61(3 B):777-81. DOI: 10.1590/S0004-282X2003000500014

16. World Health Organization. Measuring Obesity: Classification and Distribution of Anthropometric Data. Copenhagen: World Health Organization; 1989.

17. Buysse DJ, Reynolds CF 3Rd, Monk TH, Berman SR, Kupfer DJ. The Pittsburgh Sleep Quality Index: A New Instrument for Psychiatric Practice and Research. Psychiatry Res. 1989;28(2):193-213. PMID: 2748771

18. Bertolazi AN, Fagondes SC, Hoff LS, Pedro VD, Barreto SSM, Johns MW. Portuguese-language version of the Epworth sleepiness scale: validation for use in Brazil. J Bras Pneumol. 2009;35(9):877-83. PMID: 19820814

19. American College of Sports Medicine, Chodzko-Zajko WJ, Proctor DN, Fiatarone Singh MA, Minson CT, Nigg CR, Salem GJ, et al. American College of Sports Medicine position stand. Exercise and physical activity for older adults. Med Sci Sports Exerc. 2009;41(7):1510-30. PMID: 19516148

20. Borg GA. Psychophysical bases of perceived exertion. Med Sci Sports Exerc. 1982;14(5):377-81. PMID: 7154893

21. Chen MC, Liu HE, Huang HY, Chiou AF. The effect of a simple traditional exercise programme (Baduanjin exercise) on sleep quality of older adults: a randomized controlled trial. Int J Nurs Stud. 2012;49(3):265-73. PMID: 21963235

22. Sharif F, Seddigh M, Jahanbin I, Keshavarzi S. The Effect of Aerobic Exercise on Quantity and Quality of Sleep Among Elderly People Referring to Health Centers of Lar City, Southern of Iran; A Randomized Controlled Clinical Trial. Curr Aging Sci. 2015;8(3):248-55. PMID: 26537306

23. Reid KJ, Baron KG, Lu B, Naylor E. Aerobic exercise improves self-reported sleep and quality of life in older adults with insomnia. Sleep Med. 2011;11(9):934-40. PMID: 20813580

24. Chen KM, Chen MH, Chao HC, Hung HM, Lin HS, Li CH. Sleep quality, depression state, and health status of older adults after silver yoga exercises: Cluster randomized trial. Int J Nurs Stud. 2009;46:154-63. PMID: 18947826

25. Li F, Fisher KJ, Harmer P, Irbe D, Tearse RG, Weimer C. Tai chi and self-rated quality of sleep and daytime sleepiness in older adults: a randomized controlled trial. J Am Geriatr Soc. 2004;892-900. PMID: 15161452

26. Nelson ME, Layne JE, Bernstein MJ, Nuernberger A, Castaneda C, Kaliton $D$, et al. The effects of multidimensional home-based exercise on functional performance in elderly people. J Gerontol A Biol Sci Med Sci. 2004;59(2):154-60. PMID: 14999030

27. Morey MC, Snyder DC, Sloane R, Cohen HJ, Peterson B, Hartman TJ, et al. Effects of Home-Based Diet and Exercise on Functional Outcomes Among Older, Overweight Long-term Cancer Survivors. Jama. 2009;301(18):1883. PMID: 19436015

28. Ashworth NL, Chad KE, Harrison EL, Reeder BA, Marshall SC. Home versus center based physical activity programs in older adults. Cochrane Database Syst Rev. 2005;25(1):CD004017. PMID: 15674925

29. Martin KA, Sinden AR. Who will stay and who will go? A review of older adults adherence to randomized controlled trials exercise. J Aging Phys Act. 2001;9(2):91-114. DOI: 10.1123/japa.9.2.91

30. Lü J, Huang L, Wu X, Fu W, Liu Y. Effect of Tai Ji Quan training on selfreported sleep quality in elderly Chinese women with knee osteoarthritis: a randomized controlled trail. Sleep Medicine. 2017;33:70-75. PMID: 28449910

31. Gooneratne NS, Weaver TE, Cater JR, Pack FM, Arner HM, Greenberg AS, et al. Functional outcomes of excessive daytime sleepiness in older adults. J Am Geriatr Soc. 2003;51(5):642-9. PMID: 12752839

32. Lacroix A, Kressig RW, Muehlbauer T, Gschwind YJ, Pfenninger B, Bruegger $O$, et al. Effects of a supervised versus an unsupervised combined balance and strength training program on balance and muscle power in healthy older adults: A randomized controlled trial. Gerontology. 2016;62(3):27588. PMID: 26645282 